\title{
A BOUND ON THE ZERO-ERROR LIST CODING CAPACITY*
}

\author{
Erdal Arıkan \\ Department of Electrical Engineering \\ Bilkent University, Ankara 06533, Turkey
}

\begin{abstract}
We present a new bound on the zero-error list coding capacity, and using which, show that the list-of 3 capacity of the $4 / 3$ channel is at most $6 / 19$ bits, improving the best previously known bound of $3 / 8$. The relation of the bound to the graph-entropy bound of Körner and Marton is also discussed.
\end{abstract}

\section{The Bound}

Consider a discrete memoryless channel $K=(\mathcal{I}, \mathcal{J}, P)$ where $\mathcal{I}$ denotes the input alphabet, $\mathcal{J}$ the output alphabet, and $P(j \mid i)$ the probability that $j \in \mathcal{J}$ is received given that $i \in \mathcal{I}$ is transmitted. A set $S \subset \mathcal{I}^{N}$ is called independent if for every $y \in \mathcal{J}^{N}$

$$
\prod_{x \in S} \prod_{n=1}^{N} P\left(y_{n} \mid x_{n}\right)=0 \text {. }
$$

A set $\mathcal{C} \subset \mathcal{I}^{N}$ is called a zero-error list-of- $L$ code, $L \geq 1$, if every $S \subset \mathcal{C}$ with $|S|=L+1$ is an independent set. Zero-error list-of- $L$ capacity is defined by

$$
C_{L}=\limsup _{N \rightarrow \infty} \frac{1}{N} \log M(N, L)
$$

where $M(N, L)$ is the maximum possible size for a list-of- $L$ code of length $N$. (All logarithms are to to base 2.)

We call a channel $k$-uniform if $k$ is the smallest integer for which $C_{k}>0$. The new bound is as follows.

Theorem 1 The rate $R$ of any list-of-k code $\mathcal{C}$ on a $k$-uniform channel $K$ satisfies

$R-\epsilon \leq \min _{1 \leq m \leq k} \min _{x_{m+1}, \ldots, x_{k}} \min _{P^{\prime}} \frac{1}{m N} \sum_{n=1}^{N} I\left(X_{1 n}, \ldots, X_{m n} ; Y_{n} \mid x_{(m+1) n}, \ldots, x_{k n}\right)$

where $P^{\prime}$ ranges through all conditional probability assignments such that whenever $\left\{i_{1}, \ldots, i_{m}, i_{1}^{\prime}, \ldots, i_{m}^{\prime}, i_{m+1}, \ldots, i_{k}\right\}$ is independent in $K$

$$
P^{\prime}\left(j \mid i_{1}, \ldots, i_{m}, i_{m+1}, \ldots, i_{k}\right) P^{\prime}\left(j \mid i_{1}^{\prime}, \ldots, i_{m}^{\prime}, i_{m+1}, \ldots, i_{k}\right)=0
$$

for all $j$. The mutual information term is computed using the probability assignment

$$
\begin{array}{r}
P r\left\{X_{1 n}=x_{1 n}, \ldots, X_{m n}=x_{m n}, Y_{n}=y_{n}\right\}= \\
Q_{n}\left(x_{1 n}\right) \cdots Q_{n}\left(x_{m n}\right) P^{\prime}\left(y_{n} \mid x_{1 n}, \ldots, x_{k n}\right)
\end{array}
$$

where $Q_{n}$ is the empirical distribution of the nth coordinate of the codewords in $\mathcal{C}$, i.e., $Q_{n}(i)$ equals the fraction of codewords $x \in \mathcal{C}$ with $x_{n}=i$, $i \in \mathcal{I}$. The number $\epsilon$ goes to zero as $N$ increases for any fixed $R \geq 0$.
For comparison, the Körner-Marton graph-entropy bound [3] states (in the above notation) that

$$
R-\epsilon \leq \min _{m, P^{\prime}} \frac{|\mathcal{C}|^{-(k-m)}}{m N} \sum_{x_{m+1}, \ldots, x_{k}} \sum_{n=1}^{N} I\left(X_{1 n}, \ldots, X_{m n} ; Y_{n} \mid x_{(m+1) n}, \ldots, x_{k n}\right)
$$

where the outer summation is over all possible choices of distinct codewords $x_{m+1}, \ldots, x_{k} \in \mathcal{C}$. Thus, the Körner-Marton bound upperbounds the rate $R$ by (essentially) the average of the quantity $\sum_{n=1}^{N} I\left(X_{1 n}, \ldots, X_{m n} ; Y_{n} \mid x_{(m+1) n}, \ldots, x_{k n}\right)$, whereas here $R$ is bounded by the minimum of the same quantity.

The bound here may also be seen as a generalization of the Shannon bound on zero-error capacity [1], [2]. Shannon's bound is obtained by looking at the zero-error code through a single user channel; here we look at the code through a multiaccess channel.

\section{The 4/3 Channel}

The 4/3 channel has a four letter input and output alphabet $A=$ $\{0,1,2,3\}$, and the transition probabilities $P(j \mid i)=1 / 3$ for all $i, j \in A$, $i \neq j$. The bound $C_{3} \leq 6 / 19$ is obtained (after some manipulation) by applying the above theorem using the following $P^{\prime}$. (i) For any $i, i_{1}, j \in A, P^{\prime}\left(j \mid i_{1}, i, i\right)=\delta_{i j}$. (ii) For any $i_{1}, i_{2}, i_{3}, j \in A$ with $i_{2} \neq i_{3}$,

$$
P^{\prime}\left(j \mid i_{1}, i_{2}, i_{3}\right)=\left\{\begin{array}{cl}
0 & \text { if } j \in\left\{i_{1}, i_{2}, i_{3}\right\} \\
\left(4-\left|\left\{i_{1}, i_{2}, i_{3}\right\}\right|\right)^{-1} & \text { otherwise. }
\end{array}\right.
$$

\section{References}

11] C.E. Shannon, 'The zero error capacity of a noisy channel,' IEEE Trans. Inform. Theory, vol. IT-2, no. 3, pp. 8-19, 1956.

[2] P. Elias, 'Zero error capacity under list decoding,' IEEE Trans. Inform. Theory, vol. IT-34, No. 5, pp. 1070-1074,ept. 1988.

[3] J. Körner and K. Marton, 'On the capacity of uniform hypergraphs,' IEEE Trans. Inform. Theory, vol. IT-36, No.1, pp. 153-156, Jan. 1990. 